\title{
Real-time imaging of mast cell degranulation in vitro and in vivo
}

Kayo Horiguchi ${ }^{1}$, Soichiro Yoshikawa ${ }^{1, *}$, Asuka Saito ${ }^{1}$, Salma Haddad ${ }^{1}$, Takuya Ohta ${ }^{1}$, Kensuke Miyake $^{1}$, Yoshinori Yamanishi ${ }^{1}$, and Hajime Karasuyama ${ }^{1}$

${ }^{1}$ Department of Immune Regulation, Graduate School of Medical and Dental Sciences, Tokyo Medical and Dental University, Tokyo 113-8519, Japan

*Corresponding author

Soichiro Yoshikawa, Ph.D.

E-mail: yoshisou.mbch@tmd.ac.jp

Tel: +81-3-5803-5162

FAX: $+81-3-3814-7172$ 


\begin{abstract}
Abbreviations
impH, immuno-pHluorin; IgE, immunoglobulin E; TNP, 2,4,6-trinitrophenol; OVA, ovalbumin; VAMP, vesicle-associated membrane protein; SNARE, soluble N-ethylmaleimide-sensitive factor attachment protein receptor; GFP, green fluorescent protein; RFP, red fluorescent protein
\end{abstract}

\title{
Keywords
}

mast cell, degranulation, exocytosis, $\mathrm{pH}$ sensor, VAMP-8, imaging 


\section{Abstract}

Mast cells undergo degranulation in response to various stimuli and rapidly release pre-formed mediators present in secretory granules, leading to immediate-type allergic reactions. Mast cell degranulation is commonly detected and quantified in vitro by measuring histamine or $\beta$-hexosaminidase released to culture medium. However, this type of assay cannot monitor degranulation of individual cells in real time, and it is not suitable for in vivo detection of degranulation. At the aim of real time imaging of mast cell degranulation at single cell level, we here developed a fluorescent protein-based indicator of degranulation, designated immuno-pHluorin (impH). When expressed in mast cells, impH is located in the membrane of secretory granules and non-fluorescent under homeostatic conditions while it turns fluorescent following degranulation, due to the $\mathrm{pH}$ change inside of granules during exocytosis. impH enabled us to detect polarized degranulation within one single cell when mast cells were stimulated via the small area of cell surface. Transplantation of impH-expressing mast cells into mast cell-deficient mice demonstrated that impH could function as a real-time indicator of degranulation in vivo. Thus, impH is a useful tool for imaging of mast cell activation and degranulation in vitro and in vivo, and may be applied for screening of reagents regulating mast cell degranulation. 


\section{Introduction}

Mast cells are a key player in innate and acquired immune responses, particularly in allergic reactions and protective immunity against pathogens such as parasites [1, 2]. In response to various stimuli, mast cells release a series of effector molecules, including histamine, serotonin, proteases, lipid mediators, cytokines and chemokines [3-5]. Among them, histamine, serotonin and proteases are pre-formed and stored in secretory granules of mast cells, and released outside of cells rapidly after stimulation, known as degranulation, leading to immediate-type allergic reactions. The degranulation occurs by means of exocytosis of secretory granules, distinct from so-called constitutive exocytosis of secretory vesicles containing de novo synthesized cytokines and chemokines $[3,6]$. The phenomenon of mast cell degranulation has been commonly detected and quantified in vitro by measuring histamine or $\beta$-hexosaminidase released from activated mast cells [7]. While these release assays are convenient and reliable, they cannot monitor the incidence and extent of degranulation at single cell level, and are not suitable for in vivo detection of mast cell degranulation. Degranulation-associated change of mast cell morphology and protease release can be detected at single cell level by light or electron microscopic examination of tissue sections. However, this methodology cannot be applied to real-time detection of degranulation. Although recent studies have developed new methods to detect and visualize 
mast cell degranulation in real time [8-10], none of them has been applied to in vivo imaging, to our knowledge.

Exocytosis is a process of cellular secretion in which substances contained in vesicles are discharged from the cell through fusion of the vesicular membrane with the outer cell membrane $[11,12]$. This process is mediated by soluble N-ethylmaleimide-sensitive factor attachment protein receptor (SNARE) proteins which are involved in the membrane fusion [13]. Two types of SNARE exist, v-SNARE present in the vesicular membrane and t-SNARE in the plasma membrane, and docking of v-SNARE with t-SNARE leads to discharge of vesicular content. Notably, during this process, $\mathrm{pH}$ inside of vesicles shifts from low to neutral. Taking advantage of this $\mathrm{pH}$ shift, Miesenböck et al. developed a technology to visualize neurotransmission at the synapse of neurons that is mediated by exocytosis of synaptic vesicles [14]. Structure-directed combinatorial mutagenesis of green fluorescent protein (GFP) enabled to generate a $\mathrm{pH}$-sensitive mutant, designated pHluorin, that is invisible under 475-nm excitation in an environment of $\mathrm{pH}<6$ and turns fluorescent at neutral $\mathrm{pH}$. Thus, pHluorin can function as a $\mathrm{pH}$ sensor. To localize pHluorin in synaptic vesicles of neurons, pHluorin was fused to the C-terminal (luminal side) of vesicle-associated membrane protein 2 (VAMP-2) that is a member of v-SNARE and present in the membrane of synaptic vesicles [14]. This hybrid protein, designated synapto-pHluorin, was shown to be useful for visualization of exocytosis at the synapse of neurons [14]. 
In the present study, at the aim of real time imaging of mast cell degranulation at single cell level, we reconstructed synapto-pHluorin so as to optimally visualize mast cell degranulation. pHluorin was fused to VAMP-8 in place of VAMP-2 to localize the resulting protein, designated immuno-pHluorin (impH), in the secretory granule membrane of mast cells. We demonstrated that impH can be used as a useful tool for real time imaging of mast cell degranulation not only in vitro but also in vivo. 


\section{Material and Methods}

\subsection{Mice}

C57BL/6j (CLEA Japan), Kit ${ }^{\mathrm{W}-\mathrm{sh} / \mathrm{W}-\mathrm{sh}}[15]$ and Fcerl $g^{-/-}$[16] mice were maintained under specific-pathogen-free conditions in our animal facilities. All animal studies were approved by the Institutional Animal Care and Use Committee of Tokyo Medical and Dental University (Permit Number: 0150056A).

\subsection{Construction of the expression vector for immuno-pHluorin (impH)}

The Vamp8 cDNA with deletion of the stop codon was inserted into the 5 ' end of the pHluorin gene in the pCI vector (a kind gift from Dr. Gero Miesenböck, University of Oxford [14]). The VAMP-8-pHluorin fragment isolated from the pCI vector was inserted into the EcoRI and SalI sites of pIRES2-DsRed-Express2 vector (Clontech) to obtain pVAMP-8-pHluorin-IRES2-DsRed-Express2. For retrovial infection, the VAMP-8-pHluorin-IRES2-DsRed-Express2 fragment was inserted into pMSCV-IRES-GFP vector at XhoI and NotI sites to obtain pMSCV-VAMP-8-pHluorin-IRES2-DsRed-Express2.

\subsection{Preparation, transfection and activation of bone marrow-derived mast cells}

Bone marrow-derived mast cells (BMMCs) were prepared as described previously [17]. BMMCs were transfected with pVAMP-8-pHluorin-IRES2-DsRed-Express2 plasmid vector 
using the Super Electroporator NEPA21 (NEPA GENE). To establish stable transfectants,

$\begin{array}{lllll}\text { BMMCs } & \text { were } & \text { infected }\end{array}$ pMSCV-VAMP-8-pHluorin-IRES2-DsRed-Express2 or mock vector. DsRed-Express2 (RFP)-expressing cells were sorted by using FACSAria II (BD) to isolate transfectants. Purity of sorted cells was $>95 \%$. For induction of degranulation, BMMC transfectants were first sensitized with 2,4,6-trinitrophenol (TNP)-specific IgE (IGEL-b4) and then activated by incubating with $300 \mathrm{ng} / \mathrm{ml}$ of TNP-conjugated ovalbumin (TNP-OVA) or control OVA. In some experiments, IgE-sensitized BMMC transfectants were incubated with TNP-OVA-conjugated beads ( $3 \mu \mathrm{m}$ diameter) [18] that had been attached to the surface of bottom glass in culture dish by using Cell-Tak (BD).

\section{4. $\beta$-hexosaminidase release assay}

The assay was performed as described previously [18]. The percentage of degranulation was calculated using the following formula: $\% \beta$-hexosaminidase release=the activity in supernatant / the activity in supernatant plus cell lysate.

\subsection{Microscopic and flow cytometric analyses}

For staining of secretory granules, BMMC transfectants were cytospined on a slide, stained with May-Grünwald Giemsa, and examined under the light microscope. For cell surface 
staining, cells were incubated with biotinylated anti-FceRI $\alpha$ (MAR-1) (eBioscience), pacific blue-labeled anti-CD117, and APC-labeled streptavidin (Biolegend), followed by flow cytometric analysis with FACS Canto II (BD) and FlowJo (TreeStar).

\subsection{Confocal microscopic analysis}

For cytoplasmic staining, BMMC transfectants mounted on silane-coated glass slides (Muto Pure Chemicals, Tokyo, Japan) were fixed with $4 \%$ paraformaldehyde and permeabilized with ice cold methanol and $0.1 \%$ TritonX-100, followed by blocking with Protein block (Dako) containing $0.05 \%$ saponin (Sigma-Aldrich). Treated cells were incubated with mouse anti-serotonin (Dako), rabbit anti-GFP or mouse anti-GFP, rabbit anti-VAMP-3 or rabbit anti-SNAP23 (abcam), followed by staining with Alexa Fluor 555-labeled goat anti-mouse IgG or Alexa Fluor 647-labeled goat anti-rabbit IgG (Invitrogen). For staining of nucleus, DAPI (DOJINDO) was added during the secondary antibody incubation. For cell surface staining of CD63, BMMC transfectants were preincubated with unlabeled anti-CD63 (Biolegend) for masking preexisting CD63 on the cell surface. Twenty minutes after allergen stimulation, cells were stained with PE-labeled anti-CD63 (Biolegend) and anti-GFP antibodies. All samples were acquired with an A1R confocal laser scanning microscope (Nikon) and analyzed with NIS-Elements and Imaris software. 


\subsection{Real time imaging of mast cell degranulation in vitro and in vivo}

IgE-sensitized BMMC transfectants were plated on poly-L-lysine-coated dishes (MatTek) in Tyrode's buffer. Live cell imaging was performed in a humidified stage-top $\mathrm{CO}_{2}$ incubator $\left(37^{\circ} \mathrm{C}, 5 \% \mathrm{CO}_{2}\right)$, and images were recorded for $30 \mathrm{~min}$, starting one minute before the treatment with reagents $\left(300 \mathrm{ng} / \mathrm{ml}\right.$ TNP-OVA or control OVA, $50 \mathrm{mM} \mathrm{NH} \mathrm{NCl}_{4}$ or $500 \mathrm{nM}$ bafilomycin A1), by using Nikon A1R laser scanning confocal microscope. NIS-elements, Volocity and Imaris software were used for acquisition and analysis of images. For in vivo imaging of degranulation, impH-expressing BMMC transfectants derived from wild-type or Fcerl $^{-/-}$mice were transferred into $\mathrm{Kit}^{\mathrm{W}-\mathrm{sh} / \mathrm{W}-\mathrm{sh}}$ mice by intradermal injection $\left(1 \times 10^{6}\right.$ cells in $10 \mu \mathrm{l}$ PBS per injection) in the ear skin. Four weeks after mast cell reconstitution, intravital imaging of passive cutaneous anaphylaxis was performed as described previously [18]. 


\section{Results}

\subsection{Construction of a pH sensor suitable for visualization of mast cell degranulation}

Previous study reported that synapto-pHluorin could illuminate the degranulation of a rat basophil/mast cell line RBL-2H3, in addition to visualization of neurotransmission [14]. To evaluate this system in a more physiologically relevant setting, we examined the degranulation of mast cells generated from mouse bone marrow cells in culture with IL-3, known as BMMCs, in place of RBL-2H3 cells. It turned out that as many as $40 \%$ of synapto-pHluorin-expressing BMMC transfectants displayed fluorescent spots even without any stimulation (data not shown). This unexpected finding prompted us to develop a better $\mathrm{pH}$ sensor to visualize mast cell degranulation. Among the VAMP family members, mast cells express VAMP-2, 3, 7 and 8 at high levels [19, 20]. BMMCs generated from VAMP-8-deficient mice showed attenuated release of serotonin, cathepsin D, and $\beta$-hexosamidase [19], indicating the crucial contribution of VAMP-8 to the mast cell degranulation. In contrast, BMMCs derived from mice deficient for VAMP-2 or 3 appeared to degranulate normally [19]. Based on these observations, we generated a VAMP-8-containing construct, designated immuno-pHluorin (impH), in that pHluorin is fused to VAMP-8 at its C-terminal (luminal) end (Fig. 1A).

Confocal microscopic examination of impH-expressing BMMC transfectants revealed that impH stained with anti-GFP was co-localized with serotonin, but barely with 
VAMP-3 located in secretory vesicles or SNAP23 present in the plasma membrane (Fig. 1B). Thus, impH proteins were located mainly in secretory granules of BMMC transfectants, in accordance with the expression profile of native VAMP-8 [20]. May-Grünwald Giemsa staining detected no apparent anomaly in the number and size of basophilic granules in BMMC transfectants (Fig. 1C), and the level of high-affinity IgE receptor FceRI remained unaffected by the expression of impH (Fig. 1D). Moreover, when stimulated with IgE plus antigens, impH-expressing BMMC transfectants degranulated to an extent comparable to that observed in control BMMCs, as assessed by $\beta$-hexosamidase release assay (Fig. 1E). Thus, the expression of impH did not seem to alter the degranulation process in BMMCs.

\subsection{Real-time imaging of mast cell degranulation}

In a homeostatic setting, impH proteins expressed in secretory granules of BMMC transfectants were invisible (non-fluorescent) under confocal fluorescent microscopy with 488-nm excitation. When transfectants were treated with $\mathrm{NH}_{4} \mathrm{Cl}$ that increases $\mathrm{pH}$ inside of acidic vesicles including secretory granules, many green patches appeared inside of the cell within 5 min (Fig. 2A). This was also the case when transfectants were treated with bafilomycin A1 (Fig. 2A). Thus, the pH shift toward neutral in secretory granules resulted in the enhanced fluorescent intensity of $\mathrm{impH}$, suggesting the potential of $\mathrm{impH}$ as an indicator of mast cell degranulation in that the luminal $\mathrm{pH}$ in secretory granules shifts from 5.0 to 7.4 
along with exocytosis [21]. Indeed, when IgE-sensitized BMMC transfectants were stimulated with the corresponding antigen TNP-OVA, relatively large, green fluorescent patches became detectable near the cell edge within 10 min while such fluorescent patches were not detected when they were incubated with control antigen OVA (Fig. 2B). Three-dimensional, reconstructed picture showed that the vast majority of green fluorescent patches were on or close to the cell surface (Fig. 2C and Supplementary video 2). Furthermore, cell surface staining of stimulated BMMC transfectants with anti-GFP and anti-CD63 antibodies demonstrated the co-localization of impH and a typical degranulation marker CD63 (Fig. 2D). These observations suggested that the fluorescence of impH faithfully reflects the exocytosis of secretary granules, namely degranulation, in mast cells as illustrated in Fig. 2E.

\subsection{Application of impH to the study on mast cell biology}

One of the advantages using impH is that the degranulation of individual mast cells can be analyzed, in contrast to the $\beta$-hexosamidase release assay. In addition, the polarity of the degranulation event, if any, within one single cell could be detected by using the impH system. To explore this possibility, IgE-sensitized BMMC transfectants were incubated with TNP-OVA-conjugated beads with a $3 \mu \mathrm{m}$ diameter, in place of soluble TNP-OVA. Under confocal microscopy, green fluorescent patches were detected almost exclusively in the cell surface areas contacted with beads (Fig. 3), suggesting that cross-linking of FceRI molecules 
expressed on a certain part of cell surface might induce the degranulation in that area rather than the whole parts of mast cells.

The degranulation of mast cells in vivo has been indirectly demonstrated by Evans blue dye leakage elicited by mast cell-derived histamine. To explore the possible application of the impH system in vivo, we reconstituted the ear skin of mast cell-deficient Kit $^{\mathrm{W}-\mathrm{sh} / \mathrm{W}-\mathrm{sh}}$ mice with impH-expressing BMMCs generated from either wild-type or FceRI-deficient

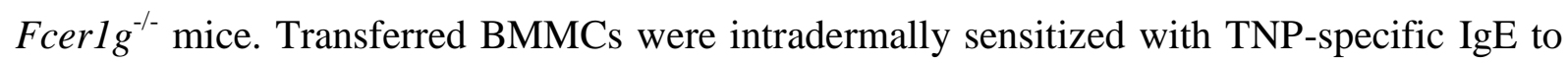
make them ready for inducing passive cutaneous anaphylaxis. Intravital imaging analysis of the ear skin detected the appearance of green fluorescent patches in wild-type but not FceRI-deficient BMMCs within 10 min after intravenous administration of TNP-OVA (Fig. 4 and Supplementary video 3), suggesting that impH can be a powerful tool to visualize mast cell degranulation in vivo. 


\section{Discussion}

Histamine or $\beta$-hexosaminidase release assay has been commonly used for measuring mast cell degranulation [11]. Although this assay is simple and convenient for evaluating degranulation of mast cells as a whole, it cannot monitor the behavior of individual cells in real time. Fluorescent dye-mediated monitoring of intracellular $\mathrm{Ca}^{2+}$ is a powerful tool for visualizing cellular activation at single cell level [9]. Nevertheless, cellular stimulation inducing $\mathrm{Ca}^{2+}$ flux does not necessarily result in mast cell degranulation. Some stimuli such as IL-33 and LPS induce cytokine production without any degranulation in mast cells [3, 22]. Therefore, the $\mathrm{Ca}^{2+}$ sensor is not an ideal indicator of mast cell degranulation. In the present study, we developed $\mathrm{impH}$, a secretory granule-resident $\mathrm{pH}$ sensor that becomes fluorescent along with mast cell degranulation. The establishment of impH enabled us to perform real time imaging of mast cell degranulation not only in vitro but also in vivo.

VAMP family members can be involved in exocytosis of both secretory granules and secretory vesicles [13]. Importantly, BMMCs derived from VAMP-8-deficient mice showed unimpaired secretion of cytokines such as $\mathrm{TNF} \alpha$, when stimulated with $\operatorname{IgE}$ plus allergens, in contrast to attenuated degranulation $[19,20]$. This is consistent with the finding that the majority of TNF $\alpha$-positive vesicles are not co-localized with VAMP-8-containing vesicles [20]. In accordance with these observations, stimulation of impH-expressing BMMCs with IL-33 or LPS induced no detectable fluorescence of impH (data not shown), in contrast to the 
IgE plus allergen stimulation. Therefore, the switching-on of impH fluorescence in activated mast cells appears to reflect exocytosis of secretory granules rather than secretory granules, faithfully reflecting degranulation.

We demonstrated in this study that impH can be used to identify the exact site of degranulation within one single cell. When IgE-sensitized mast cells were incubated with small, allergen-conjugated beads, impH fluorescence was detected almost exclusively at the mast cell-bead contact site, suggesting the polarization of degranulation determined by the site of activation signal input. This phenomenon seems reminiscent of synapse formation in $\mathrm{T}$ cell-dendritic cell or natural killer cell-target cell interface, and might play an important role in the attack of mast cells directed to pathogens such as parasites, as suggested [8]. We also demonstrated in the present study that impH can be used in vivo imaging of mast cell degranulation. Therefore, the establishment of engineered mice expressing impH only in mast cells in future study may facilitate to study such in vivo interaction of mast cells with pathogens or allergens in real time. Moreover, the impH technology could be applied for in vitro screening and in vivo evaluation of reagents that regulate mast cell degranulation. 


\section{Conflict of interest}

The authors declare no conflict of interest.

\section{Acknowledgments}

We thank Dr. Gero Miesenböck for providing the construct of pHluorin, Dr. S.J. Galli, Dr. S.

Nakae, and Dr. K. Sudo for providing Kit ${ }^{\mathrm{W}-\mathrm{sh} / \mathrm{W}-\mathrm{sh}}$ mice, and Dr. T. Takai for providing

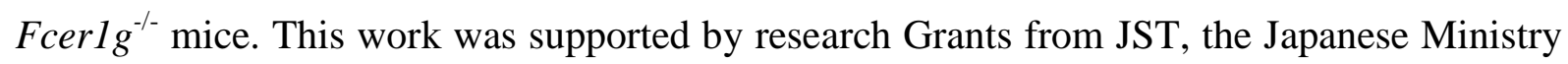
of Education, Culture, Sports, Science and Technology [15H05786] (to H. Karasuyama), and grants from Urakami foundation for Food and Food Culture Promotion (to S. Yoshikawa) and Sasakawa foundation (to K. Horiguchi). 


\section{References}

[1] S.J. Galli, M. Tsai, IgE and mast cells in allergic disease, Nat Med, 18 (2012) 693-704.

[2] S.N. Abraham, A.L. St John, Mast cell-orchestrated immunity to pathogens, Nat Rev Immunol, 10 (2010) 440-452.

[3] U. Blank, I.K. Madera-Salcedo, L. Danelli, et al., Vesicular trafficking and signaling for cytokine and chemokine secretion in mast cells, Front Immunol, 5 (2014).

[4] S. Wernersson, G. Pejler, Mast cell secretory granules: armed for battle, Nat Rev Immunol, 14 (2014) 478-494.

[5] T.C. Moon, A.D. Befus, M. Kulka, Mast cell mediators: their differential release and the secretory pathways involved, Front Immunol, 5 (2014) 569.

[6] P. Lacy, J.L. Stow, Cytokine release from innate immune cells: association with diverse membrane trafficking pathways, Blood, 118 (2011) 9-18.

[7] H.S. Kuehn, M. Radinger, A.M. Gilfillan, Measuring Mast Cell Mediator Release, Curr Protoc Immunol, CHAPTER (2010) Unit7.38-Unit37.38.

[8] R. Joulia, N. Gaudenzio, M. Rodrigues, J. et al., Mast cells form antibody-dependent degranulatory synapse for dedicated secretion and defence, Nat Commun, 6 (2015) 6174.

[9] R. Cohen, D.A. Holowka, B.A. Baird, Real-time Imaging of $\mathrm{Ca}(2+)$ Mobilization and Degranulation in Mast Cells, Methods Mol Biol (Clifton, N.J.), 1220 (2015) 347-363.

[10] H. Nishikata, C. Oliver, S.E. Mergenhagen, et al, The rat mast cell antigen AD1 
(homologue to human CD63 or melanoma antigen ME491) is expressed in other cells in culture, J Immunol, 149 (1992) 862-870.

[11] L.-G. Wu, E. Hamid, W. Shin, et al., Exocytosis and Endocytosis: Modes, Functions, and Coupling Mechanisms, Annu Rev Physiol, 76 (2014) 301-331.

[12] R.D. Burgoyne, A. Morgan, Secretory Granule Exocytosis, Physiol Rev, 83 (2003) $581-632$.

[13] A. Lorentz, A. Baumann, J. Vitte, et al., The SNARE Machinery in Mast Cell Secretion, Front Immunol, 3 (2012) 143.

[14] G. Miesenbock, D.A. De Angelis, J.E. Rothman, Visualizing secretion and synaptic transmission with pH-sensitive green fluorescent proteins, Nature, 394 (1998) 192-195.

[15] A.M. Piliponsky, C.C. Chen, M.A. Grimbaldeston, et al., Mast cell-derived TNF can exacerbate mortality during severe bacterial infections in C57BL/6-KitW-sh/W-sh mice, Am J Pathol, 176 (2010) 926-938.

[16] T. Takai, M. Li, D. Sylvestre, et al., FcR gamma chain deletion results in pleiotrophic effector cell defects, Cell, 76 (1994) 519-529.

[17] T. Ugajin, T. Kojima, K. Mukai, et al, Basophils preferentially express mouse Mast Cell Protease 11 among the mast cell tryptase family in contrast to mast cells, J Leukoc Biol, 86 (2009) 1417-1425.

[18] L. LiHua, S. Yoshikawa, T. Ohta, et al., Large particulate allergens can elicit mast 
cell-mediated anaphylaxis without exit from blood vessels as efficiently as do small soluble allergens, Biochem Biophys Res Commun, 467 (2015) 70-75.

[19] N. Puri, P.A. Roche, Mast cells possess distinct secretory granule subsets whose exocytosis is regulated by different SNARE isoforms, Proc Natl Acad Sci U S A, 105 (2008) 2580-2585.

[20] N. Tiwari, C.C. Wang, C. Brochetta, et al., VAMP-8 segregates mast cell-preformed mediator exocytosis from cytokine trafficking pathways, Blood, 111 (2008) 3665-3674.

[21] V.T.G. Schoonderwoert, G.J.M. Martens, Proton Pumping in the Secretory Pathway, J Membr Biol, 182 (2001) 159-169.

[22] L.H. Ho, T. Ohno, K. Oboki, et al., IL-33 induces IL-13 production by mouse mast cells independently of IgE-FceRI signals, J Leukoc Biol, 82 (2007) 1481-1490. 


\section{Figure legends}

Figure 1. Construction of impH and characterization of impH-expressing mast cells.

(A) Strategy of impH construction. (B) impH transfectants of BMMCs were intracellularly stained with indicated antibodies in different colors as shown. Yellow dots in merged images indicate the co-localization of green- and red-labeled proteins. (C) May-Grünwald Giemsa staining of impH or control (mock)-transfected BMMCs. Bars represent $5 \mu \mathrm{m}$. (D) FceRI expression on impH or control-transfected BMMCs. Gray histograms indicate control staining with isotype-matched antibody. (E) impH or control-transfected BMMCs were sensitized with TNP-specific IgE, then incubated for $1 \mathrm{hr}$ with TNP-OVA or control OVA, and subjected to the $\beta$-hexosaminidase release assay (mean $\pm \mathrm{SE}, \mathrm{n}=3$ ). Data shown in A-E are representative of at least three independent experiments with similar results. N.S., no significant difference.

Figure 2. Functional characterization of impH and real-time imaging of mast cell degranulation.

(A) impH-expressing BMMCs (stained in red due to DsRed expression) were treated for 30 min with $\mathrm{NH}_{4} \mathrm{Cl}$, bafilomycin A1 or control PBS, and subjected to confocal microscopic examination with 488-nm excitation. Bars represent $5 \mu \mathrm{m}$. (B and C) impH-expressing BMMCs were sensitized with TNP-specific IgE and then stimulated with TNP-OVA or control OVA. In B, live cell images were recorded under confocal fluorescent microscope at 
indicated time points. In C, 3D reconstruction of a z-stack of confocal images taken at $10 \mathrm{~min}$ post-stimulation with TNP-OVA is shown. (D) IgE-sensitized impH-expressing BMMCs were pre-treated with unlabeled anti-CD63 antibody to mask pre-existing CD63 on the cell surface, and then stimulated with TNP-OVA or control OVA. Ten minutes after stimulation, cells were fixed and stained with anti-CD63 (in red) and anti-GFP (in green) to detect newly emerged CD63 and impH, respectively, on the surface. Representative single optical sections and merged image are shown. Data shown in A-C are representative of at least three independent experiments with similar results. (E) Schematic presentation of impH localization and fluorescent status in the process of degranulation. ImpH fluorescence is quenched by the intraluminal acidic pH of secretory granules (SG). During degranulation, granules fuse with the plasma membrane, leading to exposure of the granule lumen to the neutral $\mathrm{pH}$ of extracellular environment and therefore switching on impH fluorescence.

Figure 3. Detection of polarized degranulation within one single cell in response to stimulation restricted to the small area of cell surface.

ImpH-expressing BMMCs were sensitized with TNP-specific IgE and then incubated with TNP-OVA-conjugated beads, in place of soluble TNP-OVA. Here shown are three representative images of activated cells taken under confocal microscope $10 \mathrm{~min}$ after the start of incubation. White arrows indicate TNP-OVA-conjugated beads. Data shown are 
representative of three independent experiments and a representative data is shown. Bars represent $5 \mu \mathrm{m}$.

Figure 4. Real-time imaging of mast cell degranulation in vivo.

impH-expressing wild-type (upper panels) or FcR $\gamma$-deficient (lower panels) BMMCs were subcutaneously transplanted into ears of $\mathrm{Kit}^{\mathrm{W}-\mathrm{sh} / \mathrm{W} \text {-sh }}$ mice. Recipient mice were intradermally sensitized with TNP-specific IgE, and then challenged with an intravenous injection of TNP-OVA to induce passive cutaneous anaphylaxis. Live cell images were recorded under confocal fluorescent microscope at indicated time points. Data shown are representative of three independent experiments. Bars represent $10 \mu \mathrm{m}$. 
Supplementary video 1. A time-lapse movie of the cell shown in Figure 2B.

IgE-sensitized, impH-expressing BMMC was stimulated with TNP-OVA one min after the start of recording. Real acquisition time is indicated at the top. Bar represents $5 \mu \mathrm{m}$.

Supplementary video 2. 3D reconstruction, rotated around the yz axis, of serial confocal images shown in Figure 2C.

Supplementary video 3. A time-lapse movie of the wild-type BMMCs shown in Figure 4.

Real acquisition time is indicated at the top. Bar represents $10 \mu \mathrm{m}$. 
Figure 1

A
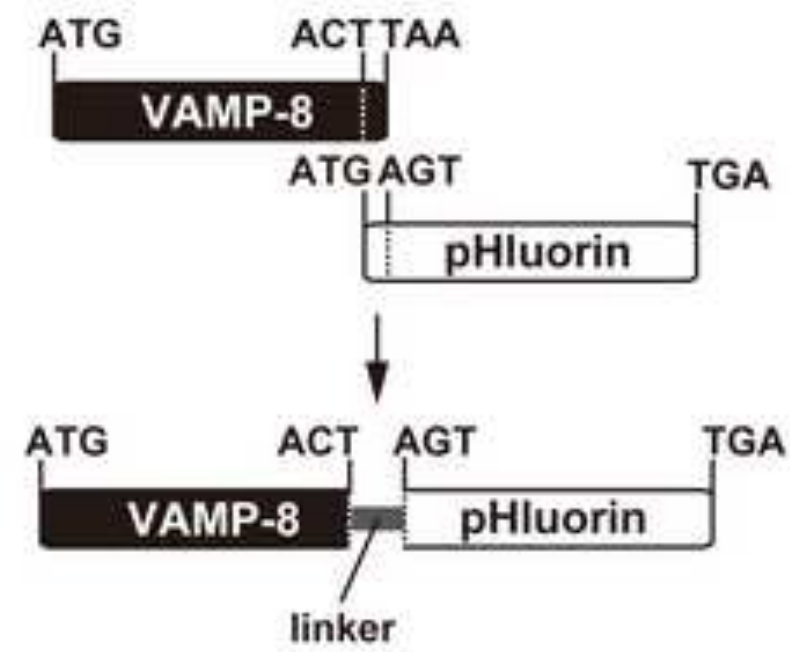

B
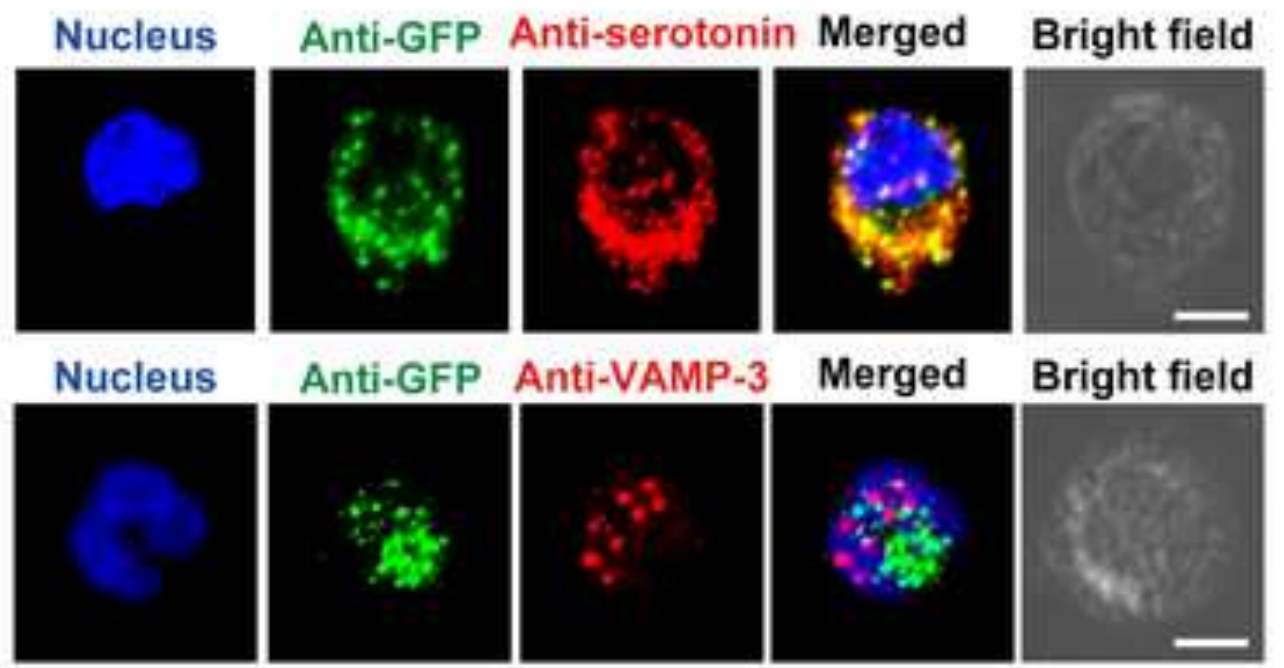

Nucleus

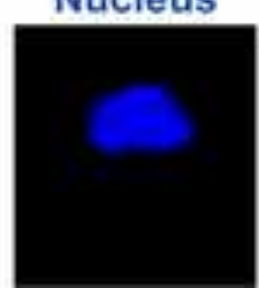

C

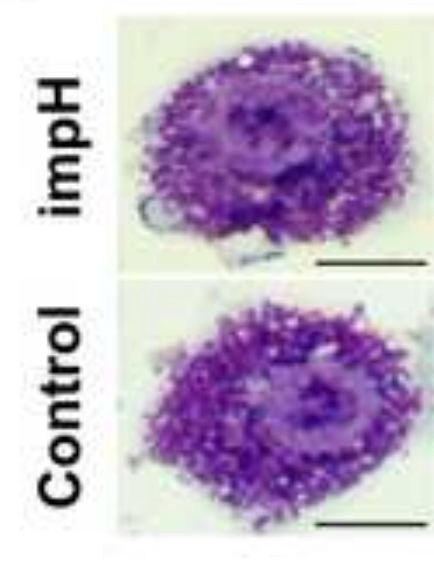

Bright field

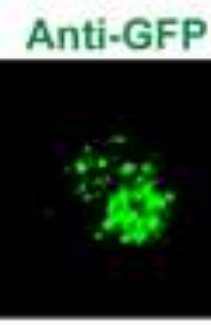

Anti-GFP

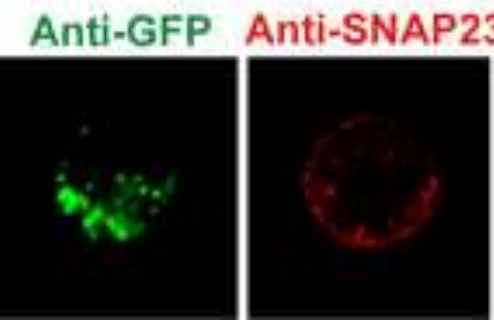

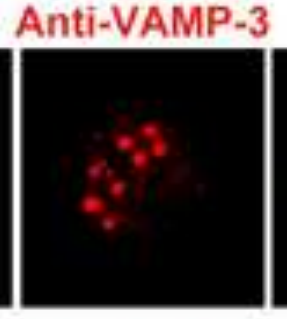
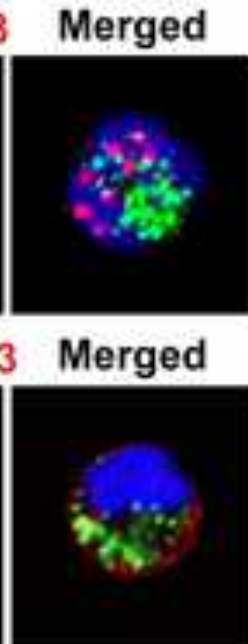

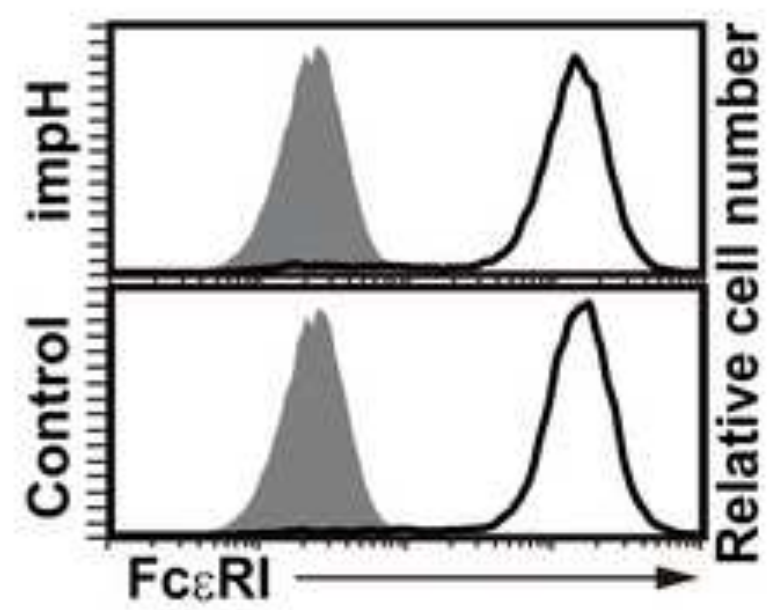

E

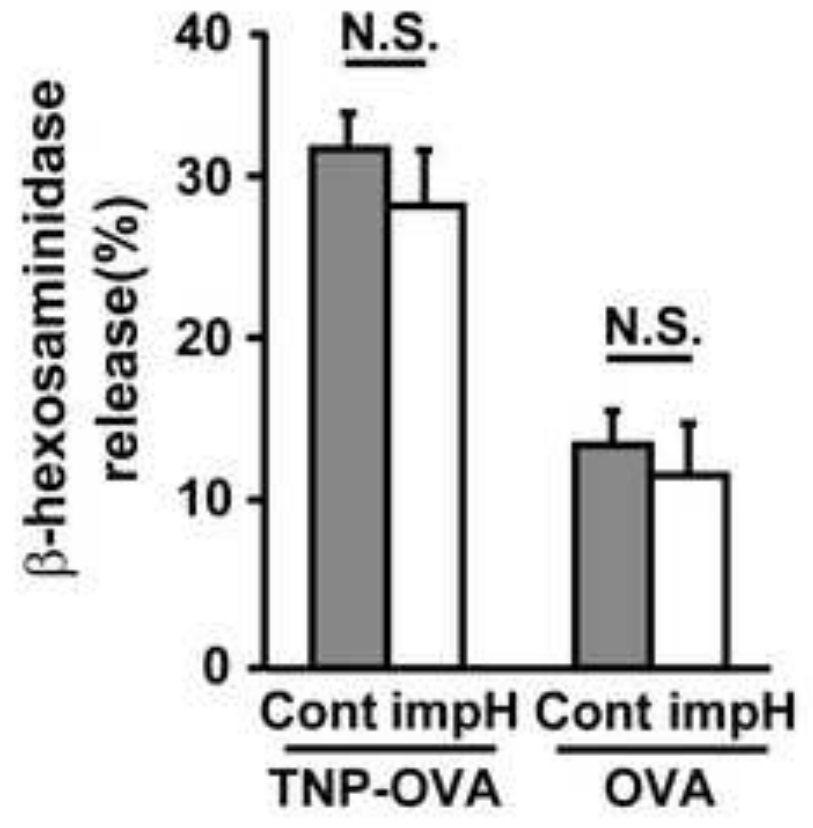


Figure 2

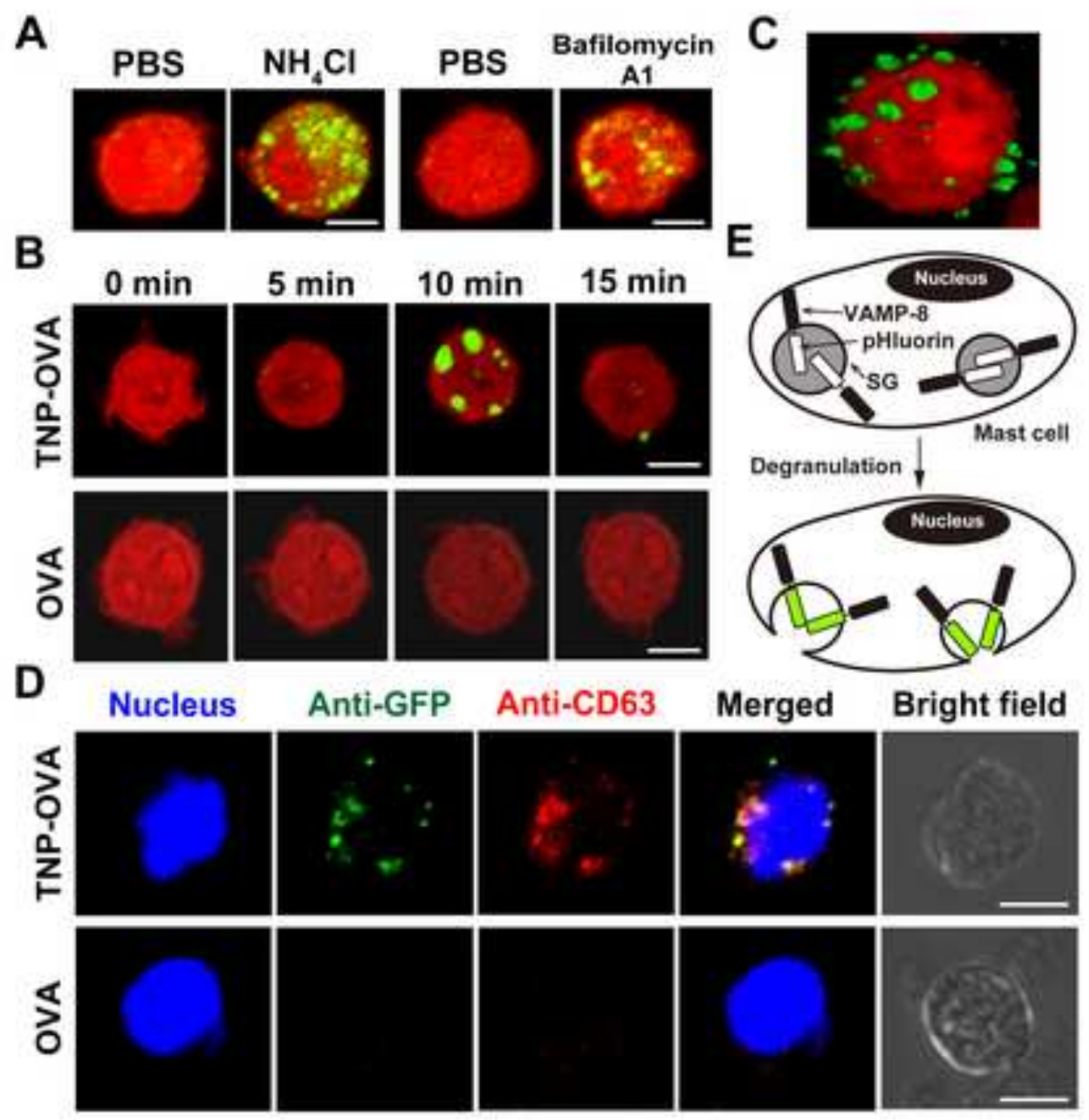


Figures

Figurs
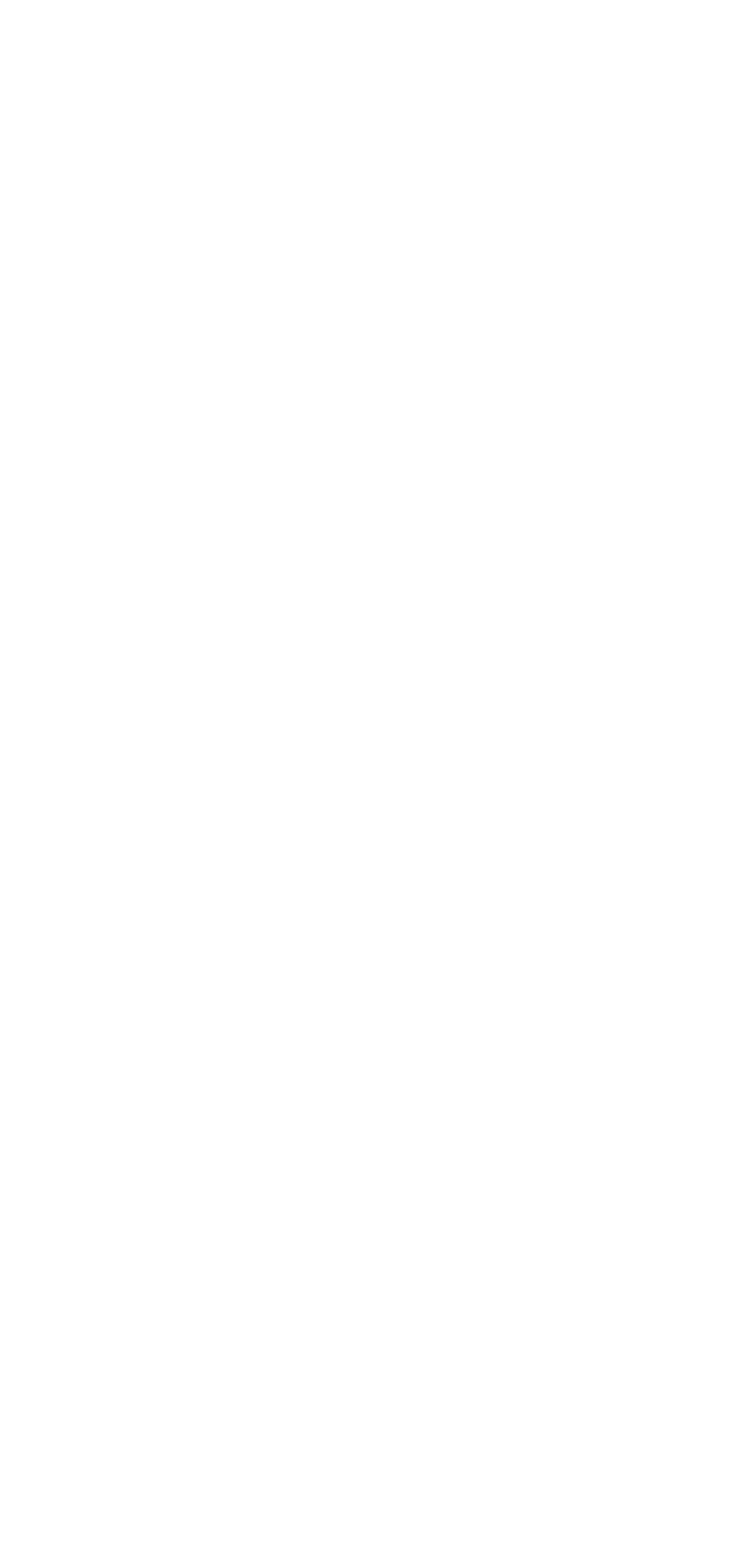

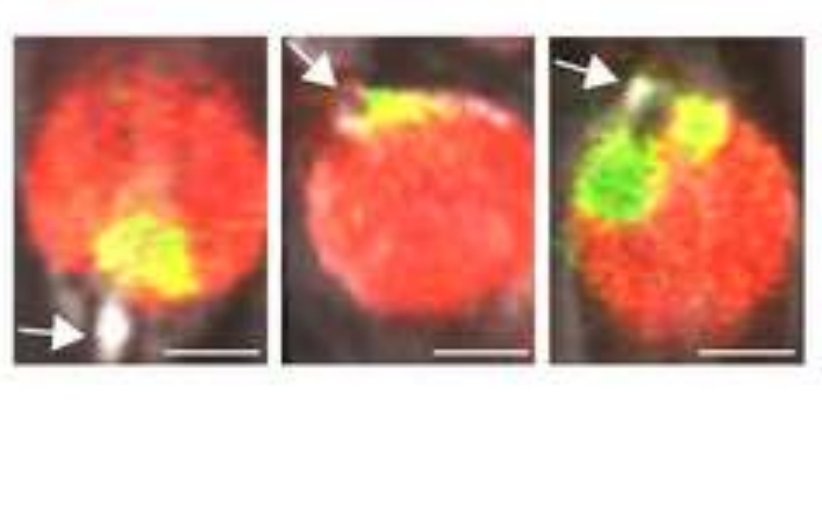 \\ .}

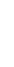

.

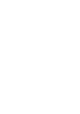

.

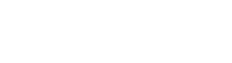

.
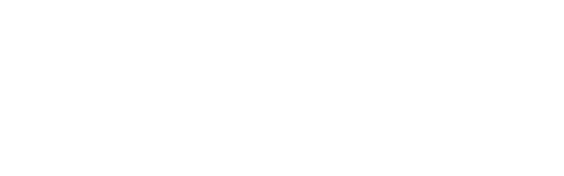

$$
\text { . }
$$$$
\text { . }
$$

(

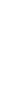

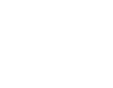
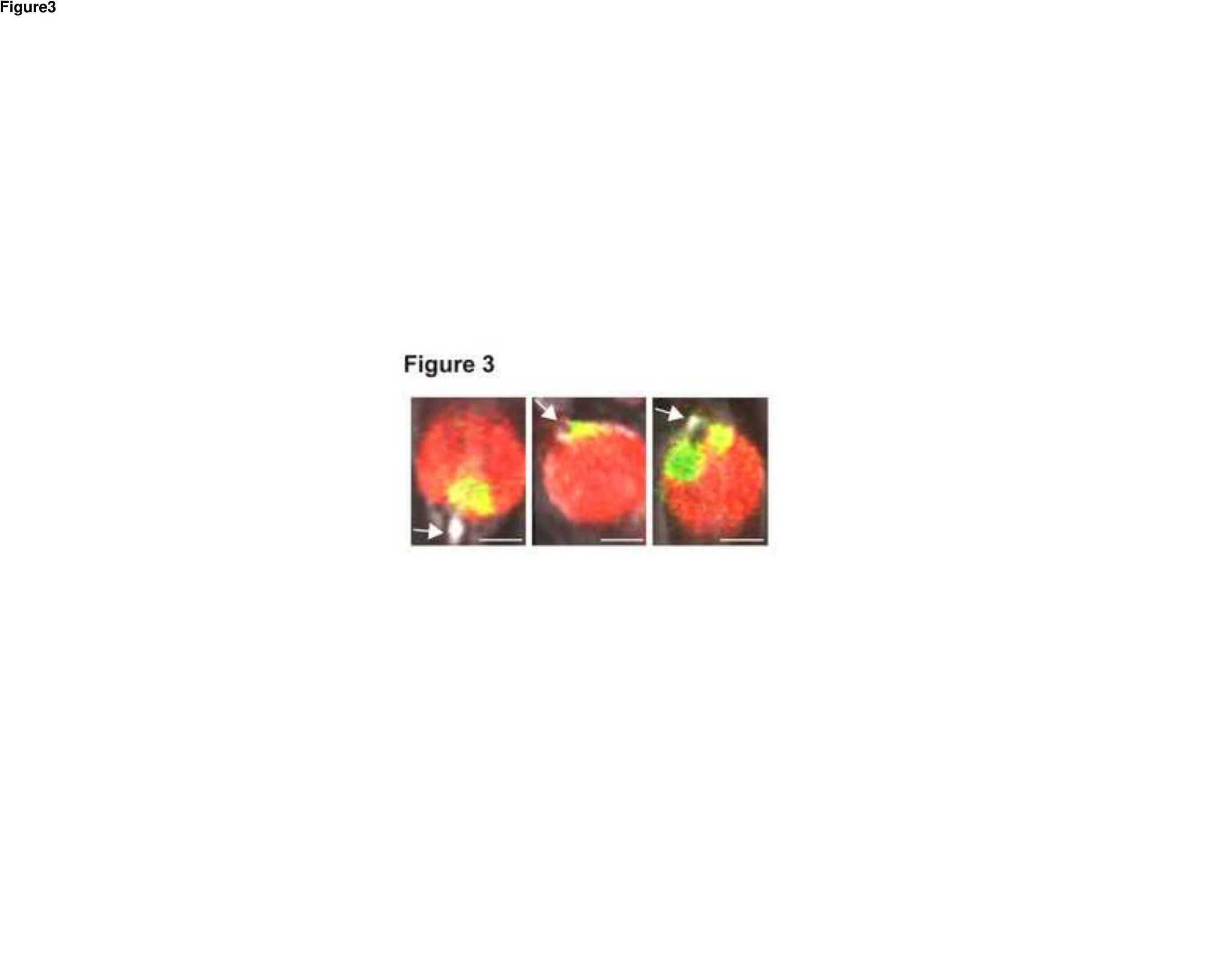

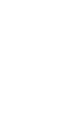

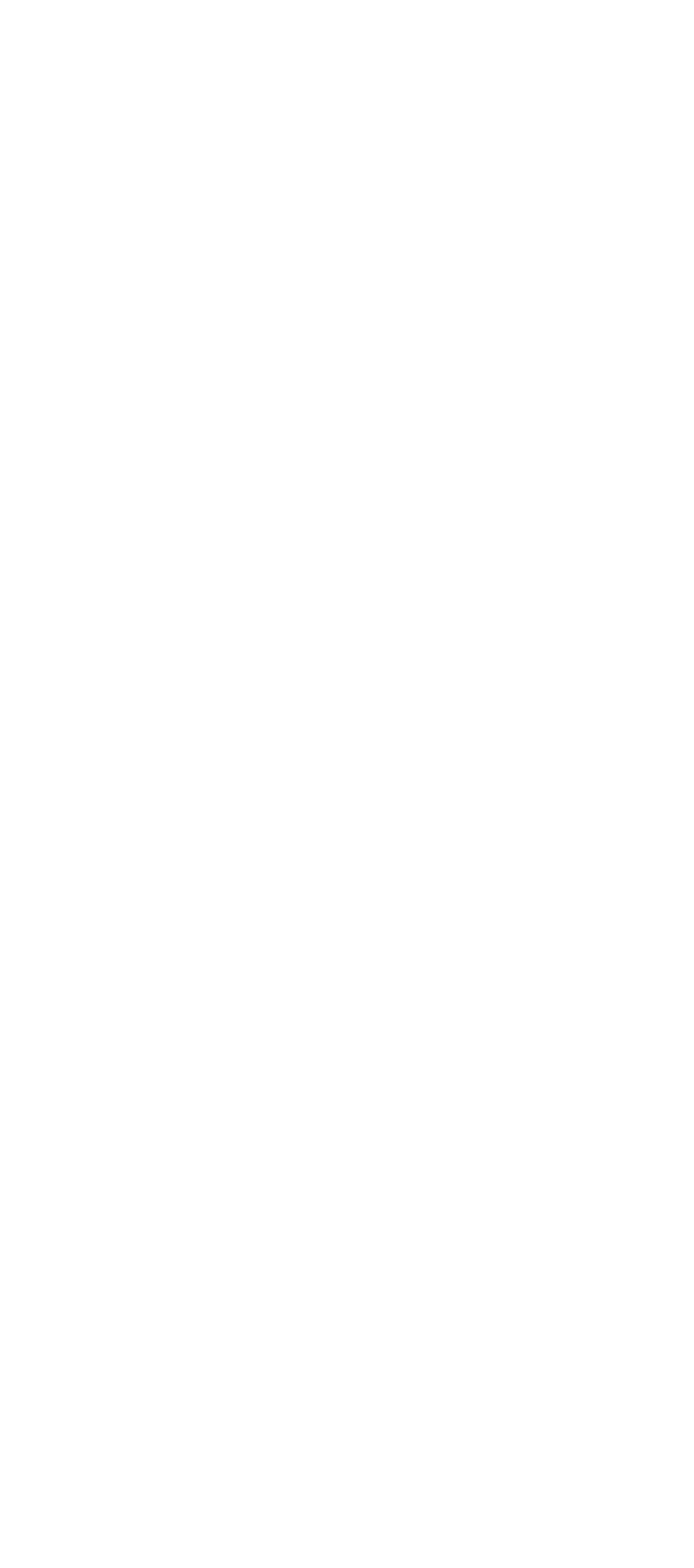

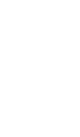

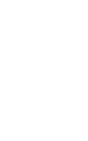


Figure 4

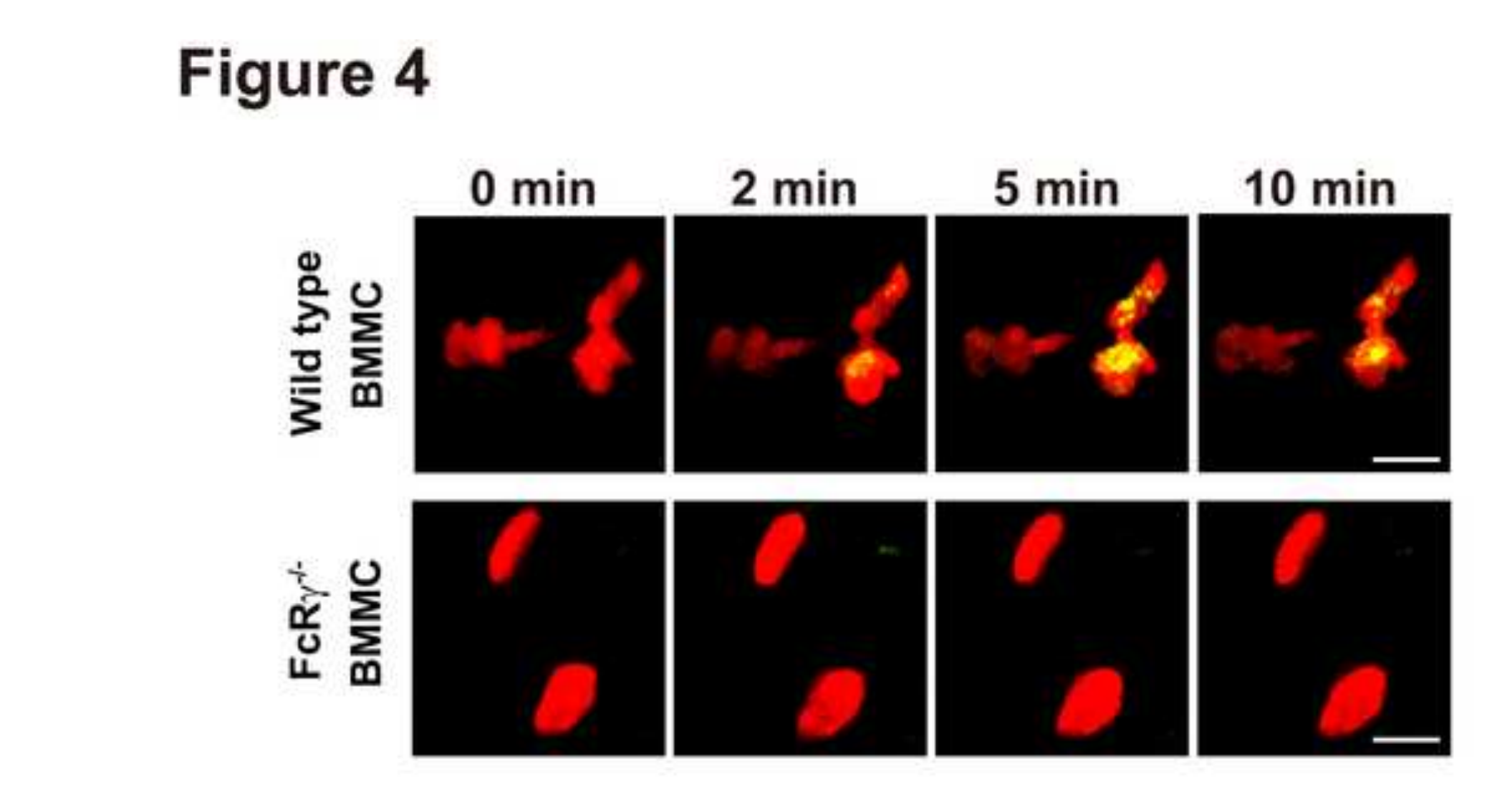

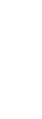
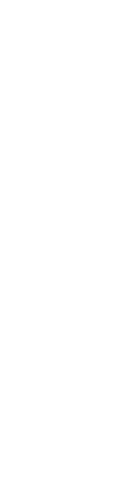